\title{
含硫醚三唑的 1,4-戊二烯-3-酮衍生物合成及生物活性研究
}

\author{
陈英李普陈梅苏时军贺军 \\ 张 敏柳立伟薛伟* \\ (贵州大学精细化工研究开发中心 绿色农药与农业生物工程国家重点实验室培育基地 \\ 教育部绿色农药与生物工程重点实验室 贵阳 550025)
}

\begin{abstract}
摘要 将含硫醇的三唑引入到 1,4-戊二烯-3-酮结构中, 合成一系列含硫醚三唑的 1,4-戊二烯-3-酮类衍生物, 其结构通 过 ${ }^{1} \mathrm{H}$ NMR、 ${ }^{13} \mathrm{C}$ NMR、HRMS 进行表征. 生物活性测试结果表明: 目标化合物对柑橘溃疡病菌 $(X$. citri)、水稻白叶枯 病菌 (X. oryzae)、烟草青枯病菌( R. solanacearum) 都表现出一定的抑制活性. 其中, 化合物 $\mathbf{F}_{\mathbf{4}} 、 \mathbf{F}_{\mathbf{6}} 、 \mathbf{F}_{\mathbf{1 6}}$ 对柑橘溃疡病菌 的 $\mathrm{EC}_{50}$ 值分别为 16.3、9.9、15.9 $\mu \mathrm{g} / \mathrm{mL}$ ，优于对照药叶枯唑 $(54.9 \mu \mathrm{g} / \mathrm{mL})$; 化合物 $\mathbf{F}_{1} 、 \mathbf{F}_{7} 、 \mathbf{F}_{15}$ 对水稻白叶枯病菌的 $\mathrm{EC}_{50}$ 值分别为 9.6、19.2、21.3 $\mu \mathrm{g} / \mathrm{mL}$ ，优于对照药叶枯唑 $(69.3 \mu \mathrm{g} / \mathrm{mL})$; 化合物 $\mathbf{F}_{3} 、 \mathbf{F}_{6}$ 对烟草青枯病菌的 $\mathrm{EC}_{50}$ 值分别为 14.2 、 $14.5 \mu \mathrm{g} / \mathrm{mL}$, 优于对照药叶枯唑 $(82.6 \mu \mathrm{g} / \mathrm{mL})$. 通过扫描电镜成像探讨了目标化合物 $\mathbf{F}_{6}$ 对柑橘溃疡病菌 $(X$. Citri $)$ 的可能 抑菌机制.
\end{abstract}

关键词 1,4-戊二烯-3-酮; 硫醚三唑; 生物活性; 扫描电镜

\section{Syntheses and Biological Activities of 1,4-Pentadien-3-one Derivatives Containing Thioether Triazole Moiety}

$$
\begin{aligned}
& \text { Chen, Ying Li, } \mathrm{Pu} \text { Chen, Mei Su, Shijun He, Jun } \\
& \text { Zhang, Min Liu, Liwei Xue, Wei* }
\end{aligned}
$$

(State Key Laboratory Breeding Base of Green Pesticide and Agricultural Bioengineering, Key Laboratory of Green Pesticide and Bioengineering of Ministry of Education, Center for Research and Development of Fine Chemicals, Guizhou University, Guiyang 550025)

\begin{abstract}
A series of novel 1,4-pentadien-3-one derivatives containing thioether triazole units were synthesized by introducing triazoles bearing thiol groups into the structures of 1,4-pentadien-3-one. The structures of the newly synthesized compounds were assigned via ${ }^{1} \mathrm{H}$ NMR, ${ }^{13} \mathrm{C}$ NMR and HRMS. Bioassays indicated that some of the compounds showed potential antibacterial activities against $X$. citri, $X$. oryzae and $R$. solanacearum. Among them, compounds $\mathbf{F}_{\mathbf{4}}, \mathbf{F}_{\mathbf{6}}$ and $\mathbf{F}_{\mathbf{1 6}}$ demonstrated appreciable inhibitory effect against Xanthomonas axonopodis pv. citri, with half-maximal effective concentration $\left(\mathrm{EC}_{50}\right)$ values of $16.3,9.9$ and $15.9 \mu \mathrm{g} / \mathrm{mL}$, which were better than commercial agent bismerthiazol $(54.9 \mu \mathrm{g} / \mathrm{mL})$. Compounds $\mathbf{F}_{1}, \mathbf{F}_{7}$ and $\mathbf{F}_{15}$ demonstrated appreciable inhibitory effects against Xanthomonas oryzae pv. Oryzae with $\mathrm{EC}_{50}$ values of 9.6, 19.2 and $21.3 \mu \mathrm{g} / \mathrm{mL}$, which were better than commercial agent bismerthiazol $(69.3 \mu \mathrm{g} / \mathrm{mL})$. Compounds $\mathbf{F}_{3}$ and $\mathbf{F}_{\mathbf{6}}$ also demonstrated appreciable inhibitory effects against Ralstonia solanacearum with $\mathrm{EC}_{50}$ values of $14.2 \mathrm{and} 14.5 \mu \mathrm{g} / \mathrm{mL}$, which were better than commercial agent bismerthiazol $(82.6 \mu \mathrm{g} / \mathrm{mL})$. The possible mechanism of the antibacterial activity of the target compound $\mathbf{F}_{\mathbf{6}}$ against Xanthomonas axonopodis was investigated through scanning electron microscopy.
\end{abstract}

Keywords 1,4-pentadien-3-one; thioether triazole; biological activity; scanning electron microscope

近年来，人类不断倡导农药向低毒、高效以及对环 境友好的方向发展，研究表明，天然活性分子及其衍生
物均有作用方式新颖、结构独特、对人畜低毒以及对环 境友好等优点, 在农药的发展历程中有着重要意义 ${ }^{[1]}$.

\footnotetext{
* Corresponding author. E-mail: wxue@gzu.edu.cn

Received March 23, 2019; revised April 18, 2019; published online June 12, 2019.

Project supported by the National Key Research and Development Program of China (No. 2017YFD0200506), and the National Natural Science Foundation of China (No. 21867003)

国家重点研发计划(No. 2017YFD0200506)、国家自然科学基金(No. 21867003)资助项目.
} 
1,4-戊二烯-3-酮类化合物是一种重要的天然产物, 具有多种生物活性, 如杀虫 ${ }^{[2,3]}$ 、抗病毒 ${ }^{[4]}$ 、抑菌 ${ }^{[5,6]}$ 、消 炎 $^{[7,8]}$ 和抗癌 ${ }^{[\rho 11]}$ 等, 因此, 该类化合物在农药领域一直 是研究的热点. 薛伟等 ${ }^{[12]}$ 将肟酯结构引入到 1,4-戊二 烯-3-酮类化合物中, 设计合成了一系列 1,4-戊二烯-3-酮 肟酯类衍生物. 生物活性测试结果表明: 在浓度为 50 $\mathrm{mg} / \mathrm{L}$ 时, 该系列化合物对小麦赤霉病菌(Gibberella)、辣 椒枯萎病菌 (Fusarium oxysporium) 和苹果腐烂病菌 (Cytospora mandshurica) 具有一定的抑制作用. 张菊平 等 ${ }^{[13]}$ 合成了一系列新型含苯并三嗪酮的 1,4-戊二烯-3酮衍生物, 并对其进行抑柑橘溃疡病菌和烟草青枯病菌 生物活性测试, 结果表明: 在药剂浓度为 $100 \mu \mathrm{g} / \mathrm{mL}$ 时, 部分化合物对上述两种病菌具有很好的抑制作用.

三氮唑杂环作为一种重要的五元含氮化合物, 在医药和农药等领域都有着广泛的应用, 如抗 癌 ${ }^{[14,15]}$ 、抗细菌 ${ }^{[16,17]}$ 、抗病毒 ${ }^{[18]}$ 、抗真菌 ${ }^{[19]}$ 和杀虫 ${ }^{[20]}$ 等, 在商品药物中三氮唑杂环类杀菌剂有: 多效唑 (Paclobutrazol)、丙环唑 (Propiconazole) 和亚胺唑 (Imibenconazole) 等 ${ }^{[21]}$. 间柏任等 ${ }^{[22]}$ 合成一系列含 1,2,4-三唑曼尼希碱的喹唑啉酮类衍生物, 生物活性测 试结果表明: 大部分化合物在 $200 \mu \mathrm{g} / \mathrm{mL}$ 浓度下, 对柑 橘溃疡病菌和水稻白叶枯病菌表现出较好的抑制活性. 林桂汕等 ${ }^{[23]}$ 设计并合成了一系列桃金娘烯醛基 2-酰基1,2,4-三唑-3-硫酮衍生物, 抑菌活性测试结果表明: 在 浓度为 $50 \mathrm{mg} / \mathrm{L}$ 时, 部分目标化合物对玉米小斑病菌和 苹果轮纹病菌表现出较好的抑菌活性.

本文利用活性拼接原理, 将含硫醇的三唑引入到 1,4-戊二烯-3-酮结构中, 合成 16 个含硫醚三唑的 1,4-戊
二烯-3-酮类化合物. 并采用浊度法测试目标化合物的 抑菌活性.

\section{1 结果与讨论}

\section{1 化合物的合成}

水合肼与二硫化碳反应得到中间体 $\mathbf{A}$, 其与醋酸反 应生成中间体 $\mathbf{B}$; 对羟基苯甲醛与丙酮反应, 通过羟醛 缩合得到中间体 $\mathbf{C}$, 其与取代醛再一次经羟醛缩合得到 中间体 $\mathbf{D}, \mathbf{D}$ 与 1,2-二溴乙烷发生取代反应生成中间体 $\mathbf{E}$ ，最后与中间体 $\mathbf{B}$ 发生取代反应得到目标化合物 $\mathbf{F}_{\mathbf{1}} \sim$ $\mathbf{F}_{16}$ (合成路线见 Scheme 1).

\section{2 化合物的结构表征}

以目标化合物 $\mathbf{F}_{3}$ 为例，对其 ${ }^{1} \mathrm{H} N \mathrm{NM} 、 \mathrm{HRMS}$ 的谱 图特点进行讨论. 在 ${ }^{1} \mathrm{H}$ NMR 谱图中, $\delta 7.75$ 出现双峰归 属于含氧苯环上 $2^{\prime}$ 的氢, $\delta 7.73$ 出现双峰归属于含氧苯 环上 $6^{\prime}$ 的氢, $\delta 7.72$ 出现双峰归属于 1,4-戊二烯-3-酤 5 位 上烯键的氢, $\delta 7.68$ 出现双峰归属于 1,4-戊二烯-3-酮 1 位上烯键的氢, $\delta 7.57$ 出现双峰归属于含 3,4-二甲基苯环 上 6 位的氢, $\delta 7.50$ 出现双峰归属于含 3,4-二甲基苯环上 5 位的氢, $\delta 7.27$ 出现单峰归属于含 3,4-二甲基苯环上 2 位的氢, $\delta 7.23$ 出现双峰归属于含氧苯环上 $3^{\prime}$ 的氢, $\delta$ 7.20 出现双峰归属于含氧苯环上 $5^{\prime}$ 的氢, $\delta 7.06$ 出现双峰 归属于 1,4-戊二烯-3-酮 2 位和 4 位上烯键的氢, $\delta 5.86$ 出现单峰归属于三唑上氨基的氢, $\delta 4.34$ 出现三峰归属 于含氧上亚甲基的氢, $\delta 3.50$ 出现三峰归属于含硫上亚 甲基的氢, $\delta 2.30$ 出现单峰归属于甲基的氢, $\delta 2.27$ 出现 单峰归属于甲基的氢, $\delta 2.26$ 出现单峰归属于甲基的氢.

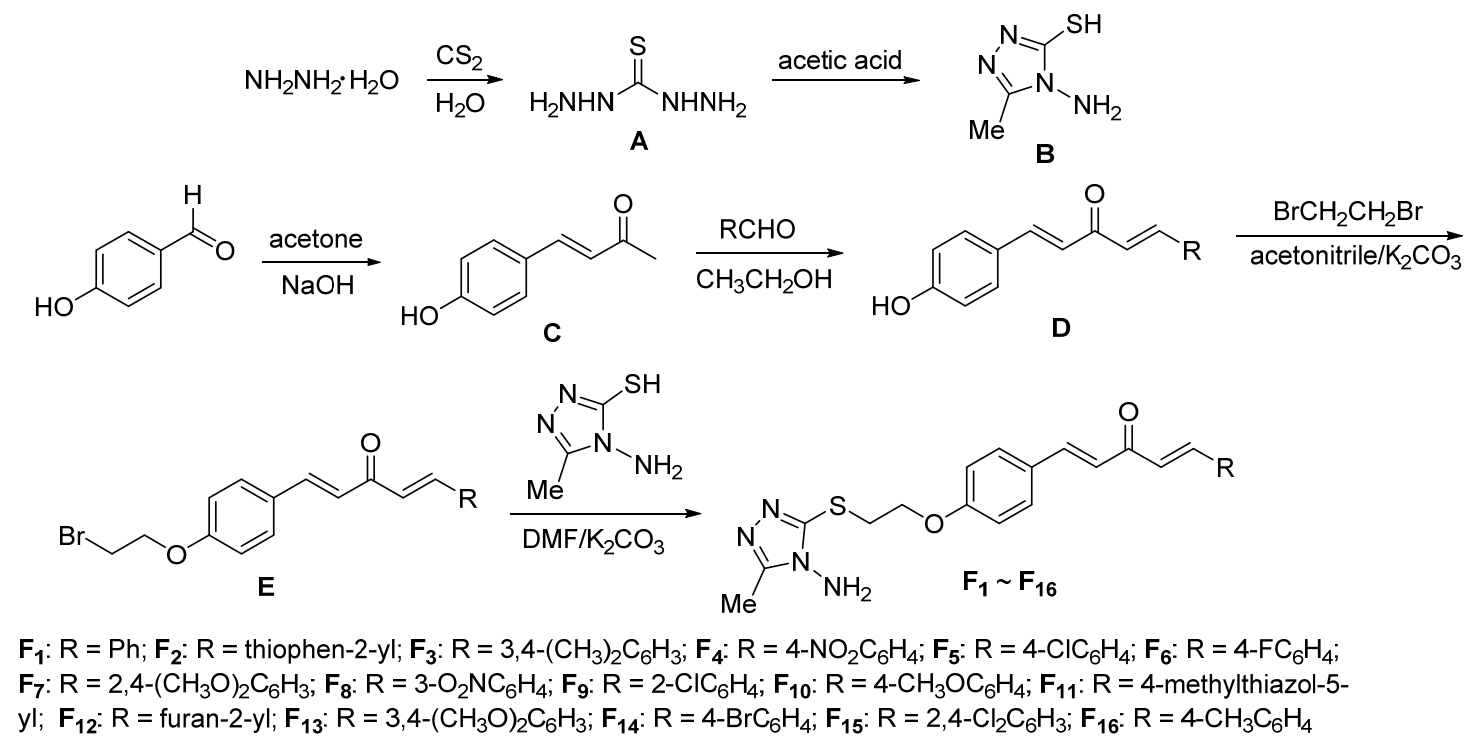

图式 1 目标化合物 $\mathbf{F}_{1} \sim \mathbf{F}_{16}$ 的合成路线

Scheme 1 Synthetic routes of target compounds $\mathbf{F}_{\mathbf{1}} \sim \mathbf{F}_{\mathbf{1 6}}$ 
在 HRMS 谱图中, 目标产物的 HRMS $[\mathrm{M}+\mathrm{H}]^{+}$实测值 和理论计算值是一致的, 误差均在 \pm 0.0030 内, 进一步 证实了目标化合物的结构.

\section{3 生物活性}

\subsection{1目标化合物的抑菌活性}

目标化合物 $\mathbf{F}_{\mathbf{1}} \sim \mathbf{F}_{16}$ 抑菌活性测试结果(表 1)表明, 所有化合物对柑橘溃疡病菌、水稻白叶枯病菌和烟草青 枯病菌均有一定的抑制活性. 其中浓度为 $100 \mu \mathrm{g} / \mathrm{mL}$ 时, 化合物 $F_{4} 、 F_{6} 、 F_{16}$ 等对柑橘溃疡病菌的抑制率超过对 照药叶枯唑 $(59.5 \%)$, 化合物 $\mathbf{F}_{1} 、 \mathbf{F}_{7} 、 \mathbf{F}_{15}$ 等对水稻白叶 枯病菌的抑制率超过对照药叶枯唑 $(56.0 \%)$, 化合物 $\mathbf{F}_{3}$ 、 $\mathbf{F}_{6}$ 对烟草青枯病菌的抑制率均超过对照药叶枯唑 $(61.1 \%)$.

\subsection{2 目标化合物的抑植物病菌活性}

以商品药叶枯唑为对照药剂, 对部分化合物进行抑 柑橘溃疡病菌、水稻白叶枯病菌和烟草青枯病菌的 $\mathrm{EC}_{50}$ 值测试，结果列于表 2 4. 由表 2 可知，所测目标化合 物的柑橘溃疡病菌 $\mathrm{EC}_{50}$ 值均优于对照药叶枯唑, 其中 化合物 $\mathbf{F}_{\mathbf{4}} 、 \mathbf{F}_{\mathbf{6}} 、 \mathbf{F}_{\mathbf{7}} 、 \mathbf{F}_{\mathbf{1 0}} 、 \mathbf{F}_{\mathbf{1 6}}$ 的 $\mathrm{EC}_{50}$ 值分别为 16.3、9.9、 $16.4 、 17.3 、 15.9 \mu \mathrm{g} / \mathrm{mL}$, 优于对照药叶枯唑 $(54.9 \mu \mathrm{g} / \mathrm{mL})$. 由表 3 可知, 所测目标化合物的水稻白叶枯病菌 $\mathrm{EC}_{50}$ 值均优于对照药叶枯唑, 其中化合物 $\mathbf{F}_{1} 、 \mathbf{F}_{7} 、 \mathbf{F}_{15}$ 的 $\mathrm{EC}_{50}$ 值分别为 9.6、19.2、21.3 $\mu \mathrm{g} / \mathrm{mL}$, 优于对照药叶枯唑 $(69.3$ $\mu \mathrm{g} / \mathrm{mL})$. 由表 4 可知, 所测目标化合物的烟草青枯病菌 $\mathrm{EC}_{50}$ 值均优于对照药叶枯唑, 其中化合物 $\mathbf{F}_{\mathbf{3}} 、 \mathbf{F}_{\mathbf{6}}$ 的 $\mathrm{EC}_{50}$ 值分别为 $14.2 、 14.5 \mu \mathrm{g} / \mathrm{mL}$, 优于对照药叶枯唑(82.6 $\mu \mathrm{g} / \mathrm{mL})$.

表 1 目标化合物 $\mathbf{F}_{\mathbf{1}} \sim \mathbf{F}_{\mathbf{1 6}}$ 的抑菌活性(抑制率/\%) $)^{a}$

Table 1 Antibacterial activities (inhibition rate/\%) of target compounds $\mathbf{F}_{\mathbf{1}} \sim \mathbf{F}_{16}$

\begin{tabular}{|c|c|c|c|c|c|c|c|}
\hline \multirow{2}{*}{ Compound } & \multirow{2}{*}{$\mathrm{R}$} & \multicolumn{2}{|c|}{ X. citri } & \multicolumn{2}{|c|}{ X. oryzae } & \multicolumn{2}{|c|}{ R. solanacearum } \\
\hline & & $100 \mu \mathrm{g} / \mathrm{mL}$ & $50 \mu \mathrm{g} / \mathrm{mL}$ & $100 \mu \mathrm{g} / \mathrm{mL}$ & $50 \mu \mathrm{g} / \mathrm{mL}$ & $100 \mu \mathrm{g} / \mathrm{mL}$ & $50 \mu \mathrm{g} / \mathrm{mL}$ \\
\hline $\mathbf{F}_{1}$ & $\mathrm{Ph}$ & $47.0 \pm 1.6$ & $36.5 \pm 4.0$ & $89.5 \pm 0.9$ & $74.3 \pm 0.1$ & $54.5 \pm 3.4$ & $49.8 \pm 5.3$ \\
\hline $\mathbf{F}_{2}$ & Thiophen-2-yl & $42.4 \pm 2.3$ & $39.0 \pm 5.0$ & $34.7 \pm 0.6$ & $1.5 \pm 0.2$ & $32.8 \pm 2.1$ & $28.3 \pm 0.1$ \\
\hline $\mathbf{F}_{3}$ & $3,4-\left(\mathrm{CH}_{3}\right)_{2} \mathrm{C}_{6} \mathrm{H}_{3}$ & $57.4 \pm 4.1$ & $41.9 \pm 2.7$ & $17.7 \pm 0.8$ & $10.6 \pm 1.4$ & $75.9 \pm 1.5$ & $62.4 \pm 0.4$ \\
\hline $\mathbf{F}_{4}$ & $4-\mathrm{NO}_{2} \mathrm{C}_{6} \mathrm{H}_{4}$ & $72.3 \pm 5.3$ & $62.8 \pm 2.8$ & $58.0 \pm 0.5$ & $41.6 \pm 1.2$ & $51.6 \pm 0.1$ & $47.9 \pm 0.5$ \\
\hline$F_{5}$ & 4- $\mathrm{ClC}_{6} \mathrm{H}_{4}$ & $68.0 \pm 0.9$ & $52.3 \pm 2.5$ & $60.5 \pm 1.1$ & $48.8 \pm 0.5$ & $48.3 \pm 2.4$ & $35.2 \pm 1.6$ \\
\hline $\mathbf{F}_{6}$ & $4-\mathrm{FC}_{6} \mathrm{H}_{4}$ & $82.5 \pm 0.2$ & $78.6 \pm 2.9$ & $59.0 \pm 4.7$ & $50.6 \pm 3.0$ & $76.8 \pm 5.4$ & $66.3 \pm 3.5$ \\
\hline $\mathbf{F}_{7}$ & $2,4-\left(\mathrm{CH}_{3} \mathrm{O}\right)_{2} \mathrm{C}_{6} \mathrm{H}_{3}$ & $71.3 \pm 0.9$ & $63.5 \pm 0.4$ & $70.0 \pm 0.4$ & $62.9 \pm 0.9$ & $53.2 \pm 0.6$ & $50.0 \pm 3.2$ \\
\hline$F_{8}$ & $3-\mathrm{NO}_{2} \mathrm{C}_{6} \mathrm{H}_{4}$ & $66.9 \pm 6.4$ & $57.1 \pm 1.1$ & $60.1 \pm 1.6$ & $51.7 \pm 1.8$ & $50.5 \pm 3.8$ & $41.7 \pm 5.2$ \\
\hline $\mathbf{F}_{\mathbf{9}}$ & $2-\mathrm{ClC}_{6} \mathrm{H}_{4}$ & $72.6 \pm 4.3$ & $64.1 \pm 2.2$ & $56.2 \pm 4.7$ & $47.6 \pm 2.6$ & $41.6 \pm 0.9$ & $35.2 \pm 0.5$ \\
\hline $\mathbf{F}_{10}$ & $4-\mathrm{CH}_{3} \mathrm{OC}_{6} \mathrm{H}_{4}$ & $70.9 \pm 2.9$ & $64.5 \pm 5.4$ & $61.7 \pm 0.9$ & $57.4 \pm 2.6$ & $48.3 \pm 2.5$ & $32.4 \pm 3.5$ \\
\hline $\mathbf{F}_{11}$ & 4-Methylthiazol-5-yl & $63.3 \pm 5.6$ & $56.5 \pm 1.2$ & $46.9 \pm 2.8$ & $44.7 \pm 0.8$ & $44.9 \pm 1.6$ & $36.3 \pm 1.3$ \\
\hline $\mathbf{F}_{12}$ & Furan-2-yl & $55.3 \pm 1.3$ & $48.1 \pm 2.5$ & $68.6 \pm 4.2$ & $56.7 \pm 0.2$ & $37.4 \pm 0.7$ & $23.2 \pm 4.8$ \\
\hline $\mathbf{F}_{13}$ & $3,4-\left(\mathrm{CH}_{3} \mathrm{O}\right)_{2} \mathrm{C}_{6} \mathrm{H}_{3}$ & $38.5 \pm 4.0$ & $25.0 \pm 1.6$ & $66.2 \pm 0.4$ & $60.4 \pm 3.9$ & $54.8 \pm 1.9$ & $42.1 \pm 1.3$ \\
\hline $\mathbf{F}_{14}$ & $4-\mathrm{BrC}_{6} \mathrm{H}_{4}$ & $73.6 \pm 6.1$ & $65.8 \pm 2.7$ & $62.2 \pm 0.7$ & $54.9 \pm 0.4$ & $50.2 \pm 0.1$ & $47.1 \pm 7.2$ \\
\hline $\mathbf{F}_{15}$ & $2,4-\mathrm{Cl}_{2} \mathrm{C}_{6} \mathrm{H}_{3}$ & $56.7 \pm 2.4$ & $45.7 \pm 4.9$ & $61.7 \pm 0.4$ & $54.9 \pm 0.6$ & $55.9 \pm 1.1$ & $42.4 \pm 0.6$ \\
\hline$F_{16}$ & 4- $\mathrm{CH}_{3} \mathrm{C}_{6} \mathrm{H}_{4}$ & $77.4 \pm 4.0$ & $68.7 \pm 1.4$ & $53.0 \pm 1.9$ & $44.6 \pm 0.6$ & $51.0 \pm 1.8$ & $33.9 \pm 2.3$ \\
\hline Bismerthiazol $^{b}$ & - & $59.5 \pm 7.7$ & $49.5 \pm 5.7$ & $56.0 \pm 1.2$ & $33.8 \pm 2.0$ & $61.1 \pm 6.2$ & $49.5 \pm 5.3$ \\
\hline
\end{tabular}

${ }^{a}$ Average of three replicates; ${ }^{b}$ The commercial bactericides (bismerthiazol) was used for comparison of antibacterial activity.

表 2 部分目标化合物对柑橘溃疡病菌的 $\mathrm{EC}_{50}$ 值 ${ }^{a}$

Table $2 \mathrm{EC}_{50}$ values of some compounds against $X$. citri

\begin{tabular}{clccc}
\hline Compound & \multicolumn{1}{c}{$\mathrm{R}$} & Toxic regression equation & $r$ & $\mathrm{EC}_{50} /\left(\mu \mathrm{g} \bullet \mathrm{mL}^{-1}\right)$ \\
\hline $\mathbf{F}_{\mathbf{4}}$ & $4-\mathrm{O}_{2} \mathrm{NC}_{6} \mathrm{H}_{4}$ & $y=0.7252 x+4.1219$ & 0.9871 & $16.3 \pm 2.5$ \\
$\mathbf{F}_{\mathbf{5}}$ & $4-\mathrm{ClC}_{6} \mathrm{H}_{4}$ & $y=0.9076 x+3.6020$ & 0.9559 & $34.7 \pm 2.3$ \\
$\mathbf{F}_{\mathbf{6}}$ & $4-\mathrm{FC}_{6} \mathrm{H}_{4}$ & $y=0.9529 x+4.0511$ & 0.9581 & $9.9 \pm 2.1$ \\
$\mathbf{F}_{\mathbf{7}}$ & $2,4-\left(\mathrm{CH}_{3} \mathrm{O}\right)_{2} \mathrm{C}_{6} \mathrm{H}_{3}$ & $y=0.6956 x+4.1554$ & 0.9889 & $16.4 \pm 1.0$ \\
$\mathbf{F}_{\mathbf{8}}$ & $3-\mathrm{O}_{2} \mathrm{NC}_{6} \mathrm{H}_{4}$ & $y=0.9427 x+3.5545$ & 0.9965 & $34.2 \pm 2.4$ \\
$\mathbf{F}_{\mathbf{9}}$ & $2-\mathrm{ClC}_{6} \mathrm{H}_{4}$ & $y=0.8344 x+3.9314$ & 0.9978 & $19.1 \pm 2.7$ \\
$\mathbf{F}_{\mathbf{1 0}}$ & $4-\mathrm{CH}_{3} \mathrm{OC}_{6} \mathrm{H}_{4}$ & $y=0.7527 x+4.0683$ & 0.9897 & $17.3 \pm 2.8$ \\
$\mathbf{F}_{\mathbf{1 4}}$ & $4-\mathrm{BrC}_{6} \mathrm{H}_{4}$ & $y=0.8865 x+3.8683$ & 0.9899 & $18.9 \pm 2.8$ \\
$\mathbf{F}_{\mathbf{1 6}}$ & $4-\mathrm{CH}_{3} \mathrm{C}_{6} \mathrm{H}_{4}$ & $y=0.9412 x+3.8669$ & 0.9965 & $15.9 \pm 1.9$ \\
Bismerthiazol $^{a}$ & - & $y=0.8357 x+3.5466$ & 0.9876 & $54.9 \pm 1.4$ \\
\hline
\end{tabular}

${ }^{a}$ The commercial bactericides (bismerthiazol) was used for comparison of antibacterial activity. 
表 3 部分目标化合物对水稻白叶枯病菌的 $\mathrm{EC}_{50}$ 值 ${ }^{a}$

Table $3 \quad \mathrm{EC}_{50}$ values of some compounds against $X$. oryzae

\begin{tabular}{clccc}
\hline Compound & \multicolumn{1}{c}{$\mathrm{R}$} & Toxic regression equation & $r$ & $\mathrm{EC} 0 /\left(\mu \mathrm{g} \bullet \mathrm{mL}^{-1}\right)$ \\
\hline $\mathbf{F}_{\mathbf{1}}$ & $\mathrm{Ph}$ & $y=1.0838 x+3.9378$ & 0.9488 & $9.6 \pm 1.9$ \\
$\mathbf{F}_{\mathbf{5}}$ & $4-\mathrm{ClC}_{6} \mathrm{H}_{4}$ & $y=0.7955 x+3.7062$ & 0.9066 & $42.3 \pm 0.5$ \\
$\mathbf{F}_{\mathbf{7}}$ & $2,4-\left(\mathrm{CH}_{3} \mathrm{O}\right)_{2} \mathrm{C}_{6} \mathrm{H}_{3}$ & $y=0.8195 x+3.9491$ & 0.9250 & $19.2 \pm 0.8$ \\
$\mathbf{F}_{\mathbf{8}}$ & $3-\mathrm{O}_{2} \mathrm{NC}_{6} \mathrm{H}_{4}$ & $y=0.8219 x+3.6715$ & 0.9099 & $41.3 \pm 1.4$ \\
$\mathbf{F}_{\mathbf{1 0}}$ & $4-\mathrm{CH}_{3} \mathrm{OC}_{6} \mathrm{H}_{4}$ & $y=0.8897 x+3.6013$ & 0.9524 & $37.3 \pm 1.5$ \\
$\mathbf{F}_{\mathbf{1 2}}$ & Furan-2-yl & $y=0.9935 x+3.5123$ & 0.9899 & $31.4 \pm 2.2$ \\
$\mathbf{F}_{\mathbf{1 3}}$ & $3,4-\left(\mathrm{CH}_{3} \mathrm{O}\right)_{2} \mathrm{C}_{6} \mathrm{H}_{3}$ & $y=0.9779 x+3.5713$ & 0.9402 & $28.9 \pm 1.4$ \\
$\mathbf{F}_{\mathbf{1 4}}$ & $4-\mathrm{BrC}_{6} \mathrm{H}_{4}$ & $y=0.8431 x+3.6698$ & 0.9886 & $37.8 \pm 0.8$ \\
$\mathbf{F}_{\mathbf{1 5}}$ & $2,4-\mathrm{Cl}_{2} \mathrm{C}_{6} \mathrm{H}_{3}$ & $y=0.4375 x+4.4190$ & 0.9756 & $21.3 \pm 0.8$ \\
Bismerthiazol $^{a}$ & - & $y=2.8364 x+0.5935$ & 0.9897 & $69.3 \pm 2.0$ \\
\hline
\end{tabular}

${ }^{a}$ The commercial bactericides (bismerthiazol) was used for comparison of antibacterial activity.

表 4 部分目标化合物对烟草青枯病菌的 $\mathrm{EC}_{50}$ 值 ${ }^{a}$

Table $4 \quad \mathrm{EC}_{50}$ values of some compounds against $R$. solanacearum

\begin{tabular}{clccc}
\hline Compound & \multicolumn{1}{c}{$\mathrm{R}$} & Toxic regression equation & $r$ & $\mathrm{EC}_{50} /\left(\mu \mathrm{g} \bullet \mathrm{mL}^{-1}\right)$ \\
\hline $\mathbf{F}_{\mathbf{3}}$ & $3,4-\left(\mathrm{CH}_{3}\right)_{2} \mathrm{C}_{6} \mathrm{H}_{3}$ & $y=0.7267 x+4.1628$ & 0.9547 & $14.2 \pm 3.3$ \\
$\mathbf{F}_{\mathbf{6}}$ & $4-\mathrm{FC}_{6} \mathrm{H}_{4}$ & $y=0.8243 x+4.0431$ & 0.9917 & $14.5 \pm 4.9$ \\
Bismerthiazol $^{a}$ & - & $y=0.9991 x+3.2599$ & 0.9399 & $82.6 \pm 0.9$ \\
\hline
\end{tabular}

${ }^{a}$ The commercial bactericides (bismerthiazol) was used for comparison of antibacterial activity.

根据表 1 4 数据可知, 大部分目标化合物具有较 好的抑菌活性. 构效关系分析表明: 当取代基团为 4- $\mathrm{NO}_{2} \mathrm{C}_{6} \mathrm{H}_{4} 、 4-\mathrm{FC}_{6} \mathrm{H}_{4} 、 4-\mathrm{CH}_{3} \mathrm{OC}_{6} \mathrm{H}_{4} 、 4-\mathrm{CH}_{3} \mathrm{C}_{6} \mathrm{H}_{4}$ 时, 目 标化合物对柑橘溃疡病菌表现出很好的抑制活性, 如 $\mathbf{F}_{\mathbf{4}} 、 \mathbf{F}_{\mathbf{6}} 、 \mathbf{F}_{\mathbf{1 0}} 、 \mathbf{F}_{\mathbf{1 6}}$ 的 $\mathrm{EC}_{50}$ 值分别为 $16.3 、 9.9 、 17.3 、 15.9$ $\mu \mathrm{g} / \mathrm{mL}$ ，均优于对照药剂叶枯唑; 当取代基团为 $\mathrm{Ph}$ 、 2,4- $\left(\mathrm{CH}_{3} \mathrm{O}\right)_{2} \mathrm{C}_{6} \mathrm{H}_{3} 、 3,4-\left(\mathrm{CH}_{3} \mathrm{O}\right)_{2} \mathrm{C}_{6} \mathrm{H}_{3} 、 2,4-\mathrm{Cl}_{2} \mathrm{C}_{6} \mathrm{H}_{3}$ 时, 目 标化合物对水稻白叶枯病菌表现出很好的抑制活性, 如 $\mathbf{F}_{\mathbf{1}} 、 \mathbf{F}_{7} 、 \mathbf{F}_{\mathbf{1 3}} 、 \mathbf{F}_{\mathbf{1 5}}$ 的 $\mathrm{EC}_{50}$ 值分别为 9.6、19.2、28.9、21.3 $\mu \mathrm{g} / \mathrm{mL}$, 均优于对照药剂叶枯唑; 当取代基团为 3,4- $\left(\mathrm{CH}_{3}\right)_{2} \mathrm{C}_{6} \mathrm{H}_{3} 、 4-\mathrm{FC}_{6} \mathrm{H}_{4}$ 时, 目标化合物对烟草青枯病 菌表现出很好的抑制活性, 如 $\mathbf{F}_{3} 、 \mathbf{F}_{6}$ 的 $\mathrm{EC}_{50}$ 值分别为 14.2、14.5 $\mu \mathrm{g} / \mathrm{mL}$ ，均优于对照药剂叶枯唑.

1.3.3 目标化合物 $\mathbf{F}_{6}$ 抑柑滳溃疡病菌 (X. citri) 作用机制 研究 ${ }^{[24]}$

通过扫描电镜观察柑橘溃疡病菌 $(X$. citri) 表面形态 实验发现, 空白对照组菌体呈杆状, 大小均一, 形貌完 整; $25 \mu \mathrm{g} / \mathrm{mL} \mathrm{F}_{6}$ 药液处理后细菌菌体两端出现细胞壁内 陷, 菌体干㾮现象; $50 \mu \mathrm{g} / \mathrm{mL} \mathrm{F}_{6}$ 药液处理后菌体细胞壁 出现不同程度的破裂, 细胞内容物外泄.

\section{2 结论}

以对羟基苯甲醛、取代醛、1,2-二溴乙烷和硫醇三 唑等为原料, 经六步反应合成 16 个新型含硫醚三唑的 1,4-戊二烯-3-酮类衍生物. 初步抑菌活性测试结果表明: 化合物对相橘溃疡病菌、水稻白叶枯病菌和烟草青枯病 菌有一定的抑制作用. 部分化合物对上述三种病菌的 (a)

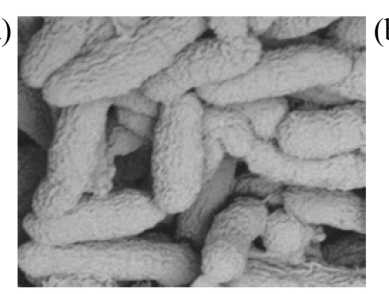

(b)

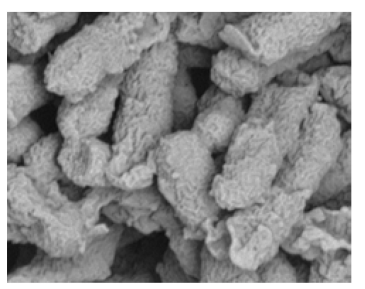

(c)

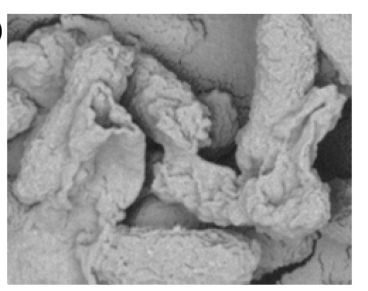

图 1 在不同浓度化合物 $\mathbf{F}_{6}$ 餒育后柑橘溃疡病菌的扫描电镜 图像

Figure 1 SEM images for $X$. citri after incubated in different concentration of compound $\mathbf{F}_{\mathbf{6}}$

(a) $c\left(\mathbf{F}_{\mathbf{6}}\right)=0 \mu \mathrm{g} / \mathrm{mL}$, (b) $c\left(\mathbf{F}_{\mathbf{6}}\right)=25 \mu \mathrm{g} / \mathrm{mL}$, (c) $c\left(\mathbf{F}_{\mathbf{6}}\right)=50 \mu \mathrm{g} / \mathrm{mL}$

$\mathrm{EC}_{50}$ 值优于对照药叶枯唑. 扫描电镜实验表明: 该类化 合物对柑橘溃疡病菌的抑菌机制是通过破坏细菌的细 胞膜结构, 导致细菌结构不完整, 从而达到抑菌的目的.

\section{3 实验部分}

\section{1 仪器与试剂}

Bruker ASCEND 400 型(400 MHz)核磁共振波谱仪 [四甲基硅烷(TMS)为内标, 瑞士 Bruker 公司]; Thermo Scientic Q Exactive 高分辨质谱仪(HRMS, 赛默飞世尔 科技有限公司); X-4 型数字显微熔点测定仪(北京泰克 
仪器有限公司); Sartorius 电子天平(德国赛多利斯集团); QY-20 型三用紫外分析仪(上海市安亭电子仪器厂). 1,2二溴乙烷和取代醛购于上海泰坦科技股份有限公司; 水 合肼、二硫化碳等试剂均为市售分析纯. 中间体 $\mathbf{A} 、 \mathbf{B}$

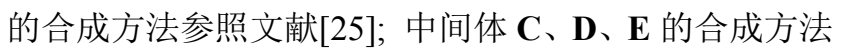
参照文献[26, 27].

\section{2 目标化合物 $F_{1} \sim F_{16}$ 的合成}

在 $100 \mathrm{~mL}$ 的单口圆底烧瓶中加入 $0.5 \mathrm{~g}$ 中间体 $\mathbf{B}$ (3.8 mmol)、1.3 $\mathrm{g} \mathrm{K}_{2} \mathrm{CO}_{3}$ (9.6 mmol)和 $60 \mathrm{~mL} N, N$-二甲基 甲酰胺(DMF), 常温搅拌 $0.5 \mathrm{~h}$, 然后缓慢加入 $1.1 \mathrm{~g}$ 中间 体 E (3.2 mmol) 的 DMF 溶液, 继续搅拌 $6 \sim 8 \mathrm{~h}$. 薄层色 谱(TLC)跟踪反应, 当反应结束, 停止反应, 反应液用 $100 \mathrm{~mL}$ 的水分散, 乙酸乙酯萃取 $(25 \mathrm{~mL})$, 合并有机层, 用饱和食盐水洗涤 $(40 \mathrm{~mL} \times 3)$ 、无水硫酸钠干燥、减压 除去溶剂, 得粗产物, 经柱层析提纯得到目标化合物 $\mathbf{F}_{1} \sim \mathbf{F}_{16}$.

1-(4-(2-((4-氨基-5-甲基-4H-1,2,4-三唑-3-基)硫基) 乙氧基)苯基)-5-苯基-1,4-戊二烯-3-酮 $\left(\mathbf{F}_{1}\right)$ : 黄色固体, 收率 60\%. m.p. 130.2 131.4 ${ }^{\circ} \mathrm{C} ;{ }^{1} \mathrm{H}$ NMR (400 MHz, DMSO- $\left.d_{6}\right) \delta: 7.80(\mathrm{~d}, J=6.3 \mathrm{~Hz}, 2 \mathrm{H}), 7.76(\mathrm{~d}, J=5.8 \mathrm{~Hz}$, 2H), 7.72 (t, $J=5.8 \mathrm{~Hz}, 2 \mathrm{H}), 7.46$ (t, $J=6.8 \mathrm{~Hz}, 1 \mathrm{H}), 7.42$ (t, $J=5.5 \mathrm{~Hz}, 2 \mathrm{H}), 7.31(\mathrm{~d}, J=7.0 \mathrm{~Hz}, 1 \mathrm{H}), 7.20$ (d, $J=7.1$ $\mathrm{Hz}, 1 \mathrm{H}), 7.07$ (d, J=7.6 Hz, 1H), 7.02 (d, $J=7.9 \mathrm{~Hz}, 1 \mathrm{H})$, $5.87(\mathrm{~s}, 2 \mathrm{H}), 4.32(\mathrm{t}, J=6.3 \mathrm{~Hz}, 2 \mathrm{H}), 3.49(\mathrm{t}, J=6.3 \mathrm{~Hz}$, 2H), $2.28(\mathrm{~s}, 3 \mathrm{H}) ;{ }^{13} \mathrm{C}$ NMR (100 MHz, DMSO-d $) \delta$ : $188.82,160.58,153.87,151.15,143.16,142.74,135.28$, $130.95,130.89,129.45,128.97,128.05,126.25,124.01$, 115.46, 66.82, 30.11, 10.25; ESI-HRMS calcd for $\mathrm{C}_{22} \mathrm{H}_{23} \mathrm{O}_{2} \mathrm{~N}_{4} \mathrm{~S}[\mathrm{M}+\mathrm{H}]^{+}$407.15362, found 407.15302.

1-(4-(2-((4-氨基-5-甲基-4H-1,2,4-三唑-3-基)硫基) 乙氧基)苯基)-5-(噻吩-2-基)-1,4-戊二烯-3-酮( $\left.\mathbf{F}_{2}\right)$ : 黄色 固体, 收率 51\%. m.p. 147.4 148.8 ${ }^{\circ} \mathrm{C} ;{ }^{1} \mathrm{H}$ NMR (400 MHz, DMSO- $\left.d_{6}\right) \delta: 7.95(\mathrm{~d}, J=5.8 \mathrm{~Hz}, 1 \mathrm{H}), 7.77(\mathrm{~d}, J=$ $5.9 \mathrm{~Hz}, 1 \mathrm{H}), 7.75$ (d, $J=5.5 \mathrm{~Hz}, 1 \mathrm{H}), 7.74(\mathrm{~d}, J=5.8 \mathrm{~Hz}$, 1H), 7.69 (d, $J=5.9 \mathrm{~Hz}, 1 \mathrm{H}), 7.59$ (d, $J=6.4 \mathrm{~Hz}, 1 \mathrm{H}), 7.25$ $(\mathrm{d}, J=5.9 \mathrm{~Hz}, 1 \mathrm{H}), 7.19$ (t, $J=7.5 \mathrm{~Hz}, 1 \mathrm{H}), 7.05$ (d, $J=7.2$ $\mathrm{Hz}, 2 \mathrm{H}), 6.96$ (d, $J=6.8 \mathrm{~Hz}, 1 \mathrm{H}), 5.86(\mathrm{~s}, 2 \mathrm{H}), 4.34$ (t, $J=$ $6.4 \mathrm{~Hz}, 2 \mathrm{H}), 3.50(\mathrm{t}, J=6.3 \mathrm{~Hz}, 2 \mathrm{H}), 2.29(\mathrm{~s}, 3 \mathrm{H}) ;{ }^{13} \mathrm{C}$ NMR $\left(100 \mathrm{MHz}, \mathrm{DMSO}-d_{6}\right) \delta: 188.12,160.55,153.84$, $151.11,142.75,140.34,135.69,132.86,130.95,130.51$, $129.21,128.08,125.23,123.79,115.44,66.84,30.16$, 10.24; ESI-HRMS calcd for $\mathrm{C}_{20} \mathrm{H}_{21} \mathrm{O}_{2} \mathrm{~N}_{4} \mathrm{~S}_{2} \quad[\mathrm{M}+\mathrm{H}]^{+}$ 413.11004, found 413.10922.

1-(4-(2-((4-氨基-5-甲基-4H-1,2,4-三唑-3-基)硫基) 乙氧基)苯基)-5-(3,4-二甲基苯基)-1,4-戊二烯-3-酮 $\left(\mathbf{F}_{3}\right)$ :
黄色固体，收率 34\%. m.p. 90.2 91.8 ${ }^{\circ} \mathrm{C} ;{ }^{1} \mathrm{H}$ NMR (400 MHz, DMSO-d $) \delta: 7.75(\mathrm{~d}, J=6.1 \mathrm{~Hz}, 1 \mathrm{H}), 7.73$ (d, $J=$ $5.4 \mathrm{~Hz}, 1 \mathrm{H}), 7.72(\mathrm{~d}, J=6.6 \mathrm{~Hz}, 1 \mathrm{H}), 7.68(\mathrm{~d}, J=5.9 \mathrm{~Hz}$, $1 \mathrm{H}), 7.57$ (d, $J=6.4 \mathrm{~Hz}, 1 \mathrm{H}), 7.50$ (d, $J=6.8 \mathrm{~Hz}, 1 \mathrm{H}), 7.27$ $(\mathrm{s}, 1 \mathrm{H}), 7.23(\mathrm{~d}, J=5.8 \mathrm{~Hz}, 1 \mathrm{H}), 7.20(\mathrm{~d}, J=7.0 \mathrm{~Hz}, 1 \mathrm{H})$, $7.06(\mathrm{~d}, J=7.8 \mathrm{~Hz}, 2 \mathrm{H}), 5.86(\mathrm{~s}, 2 \mathrm{H}), 4.34(\mathrm{t}, J=6.4 \mathrm{~Hz}$, 2H), 3.50 (t, $J=6.4 \mathrm{~Hz}, 2 \mathrm{H}), 2.30(\mathrm{~s}, 3 \mathrm{H}), 2.27$ (s, 3H), $2.26(\mathrm{~s}, 3 \mathrm{H}) ;{ }^{13} \mathrm{C}$ NMR $\left(100 \mathrm{MHz}, \mathrm{DMSO}-d_{6}\right) \delta: 188.76$, $160.52,153.84,151.09,143.00,142.77,139.78,137.36$, $132.87,130.87,130.56,129.89,128.12,126.68,125.19$, $124.13, \quad 115.48, \quad 66.87, \quad 30.22, \quad 19.91, \quad 19.79, \quad 10.23$; ESI-HRMS calcd for $\mathrm{C}_{24} \mathrm{H}_{27} \mathrm{O}_{2} \mathrm{~N}_{4} \mathrm{~S}[\mathrm{M}+\mathrm{H}]^{+}$435.18492, found 435.18408 .

1-(4-(2-((4-氨基-5-甲基-4H-1,2,4-三唑-3-基)硫基) 乙氧基)苯基)-5-(4-硝基苯基)-1,4-戊二烯-3-酮( $\left.\mathbf{F}_{4}\right)$ : 黄色 固体, 收率: $34 \%$. m.p. 137.2 138.5 ${ }^{\circ} \mathrm{C} ;{ }^{1} \mathrm{H}$ NMR (400 $\left.\mathrm{MHz}, \mathrm{DMSO}-d_{6}\right) \delta: 8.33(\mathrm{~d}, J=7.5 \mathrm{~Hz}, 2 \mathrm{H}), 8.10(\mathrm{~d}, J=$ $7.3 \mathrm{~Hz}, 2 \mathrm{H}), 7.87$ (d, $J=6.0 \mathrm{~Hz}, 2 \mathrm{H}), 7.80$ (d, $J=6.8 \mathrm{~Hz}$, 2H), 7.59 (d, $J=6.4 \mathrm{~Hz}, 1 \mathrm{H}), 7.26$ (d, $J=6.0 \mathrm{~Hz}, 1 \mathrm{H}), 7.11$ (d, $J=7.8 \mathrm{~Hz}, 2 \mathrm{H}), 5.90$ (s, 2H), 4.38 (t, $J=6.3 \mathrm{~Hz}, 2 \mathrm{H})$, $3.54(\mathrm{t}, J=6.4 \mathrm{~Hz}, 2 \mathrm{H}), 2.33(\mathrm{~s}, 3 \mathrm{H}) ;{ }^{13} \mathrm{C} \mathrm{NMR}(100 \mathrm{MHz}$, DMSO- $\left.d_{6}\right) \delta: 188.68,160.77,153.85,151.11,148.43$, $144.05,141.89,139.92,131.07,129.94,129.80,127.93$, $124.51,124.02,115.53,66.87,30.14,10.24$; ESI-HRMS calcd for $\mathrm{C}_{22} \mathrm{H}_{22} \mathrm{O}_{4} \mathrm{~N}_{5} \mathrm{~S}[\mathrm{M}+\mathrm{H}]^{+}$452.13870, found 452.13791 .

1-(4-(2-((4-氨基-5-甲基-4H-1,2,4-三唑-3-基)硫基) 乙氧基)苯基)-5-(4-氯苯基)-1,4-戊二烯-3-酮 $\left(\mathbf{F}_{5}\right)$ : 黄色固 体, 收率: 54\%. m.p. 165.8 167.1 ${ }^{\circ} \mathrm{C} ;{ }^{1} \mathrm{H}$ NMR (400 MHz, DMSO- $\left.d_{6}\right) \delta: 7.84(\mathrm{~d}, J=6.6 \mathrm{~Hz}, 2 \mathrm{H}), 7.78(\mathrm{~d}, J=$ $6.1 \mathrm{~Hz}, 2 \mathrm{H}), 7.75(\mathrm{~d}, J=6.0 \mathrm{~Hz}, 1 \mathrm{H}), 7.54$ (d, $J=6.5 \mathrm{~Hz}$, 2H), 7.39 (d, $J=6.1 \mathrm{~Hz}, 1 \mathrm{H}), 7.22$ (d, $J=6.0 \mathrm{~Hz}, 1 \mathrm{H}), 7.08$ (d, $J=7.9 \mathrm{~Hz}, 2 \mathrm{H}), 7.04$ (d, $J=7.7 \mathrm{~Hz}, 1 \mathrm{H}), 5.89$ (s, 2H), $4.36(\mathrm{t}, J=6.4 \mathrm{~Hz}, 2 \mathrm{H}), 3.52(\mathrm{t}, J=6.3 \mathrm{~Hz}, 2 \mathrm{H}), 2.31(\mathrm{~s}$, $3 \mathrm{H}) ;{ }^{13} \mathrm{C}$ NMR (100 MHz, DMSO- $\left.d_{6}\right) \delta 188.74,160.62$, $153.85,151.12,143.36,141.25,135.33,134.27,130.95$, $130.64,129.49,128.01,126.82,124.06,115.49,66.84$, 30.14, 10.24; ESI-HRMS calcd for $\mathrm{C}_{22} \mathrm{H}_{22} \mathrm{O}_{2} \mathrm{~N}_{4} \mathrm{ClS}$ [M+ $\mathrm{H}]^{+}$441.11465, found 441.11404.

1-(4-(2-((4-氨基-5-甲基-4H-1,2,4-三唑-3-基)硫基) 乙氧基)苯基)-5-(4-氟苯基)-1,4-戊二烯-3-酮 $\left(\mathbf{F}_{6}\right)$ : 黄色固 体, 收率: 50\%. m.p. 122.5 123.7 ${ }^{\circ} \mathrm{C} ;{ }^{1} \mathrm{H}$ NMR (400 $\left.\mathrm{MHz}, \mathrm{DMSO}-d_{6}\right) \delta: 7.87(\mathrm{~d}, J=6.0 \mathrm{~Hz}, 1 \mathrm{H}), 7.85$ (d, $J=$ $6.9 \mathrm{~Hz}, 1 \mathrm{H}), 7.80(\mathrm{~d}, J=6.8 \mathrm{~Hz}, 1 \mathrm{H}), 7.75(\mathrm{~d}, J=6.0 \mathrm{~Hz}$, $3 \mathrm{H}), 7.34$ (d, $J=6.9 \mathrm{~Hz}, 1 \mathrm{H}), 7.30$ (d, $J=6.2 \mathrm{~Hz}, 2 \mathrm{H}), 7.20$ 
(d, $J=7.0 \mathrm{~Hz}, 1 \mathrm{H}), 7.06$ (d, $J=7.9 \mathrm{~Hz}, 2 \mathrm{H}), 5.86$ (s, 2H), 4.34 (t, $J=6.4 \mathrm{~Hz}, 2 \mathrm{H}), 3.51$ (t, $J=6.3 \mathrm{~Hz}, 2 \mathrm{H}), 2.30$ (s, $3 \mathrm{H}) ;{ }^{13} \mathrm{C}$ NMR (100 MHz, DMSO- $\left.d_{6}\right) \delta: 188.75,160.59$ (d, $J=247.0 \mathrm{~Hz}), 153.84,151.09,143.15,141.49,131.98$, $131.29,130.90,128.06,126.13,124.07,116.58,116.36$, $115.50,66.88,30.21,10.24$; ESI-HRMS calcd for $\mathrm{C}_{22} \mathrm{H}_{22}-$ $\mathrm{O}_{2} \mathrm{~N}_{4} \mathrm{FS}[\mathrm{M}+\mathrm{H}]^{+} 425.14420$, found 425.14322 .

1-(4-(2-((4-氨基-5-甲基-4H-1,2,4-三唑-3-基)硫基) 乙氧基)苯基)-5-(2,4-二甲氧基苯基)-1,4-戊二烯-3-酮 $\left(\mathbf{F}_{7}\right)$ : 黄色固体, 收率: 31\%. m.p. 132.5 134.1 ${ }^{\circ} \mathrm{C} ;{ }^{1} \mathrm{H}$ NMR (400 MHz, DMSO- $\left.d_{6}\right) \delta: 7.89(\mathrm{~d}, J=6.9 \mathrm{~Hz}, 1 \mathrm{H})$, $7.73(\mathrm{~d}, J=6.4 \mathrm{~Hz}, 2 \mathrm{H}), 7.67(\mathrm{~d}, J=6.0 \mathrm{~Hz}, 1 \mathrm{H}), 7.58$ (d, $J=6.4 \mathrm{~Hz}, 1 \mathrm{H}), 7.24$ (d, $J=6.0 \mathrm{~Hz}, 1 \mathrm{H}), 7.15$ (d, $J=6.9$ $\mathrm{Hz}, 1 \mathrm{H}), 7.08$ (d, $J=7.9 \mathrm{~Hz}, 1 \mathrm{H}), 7.04$ (s, 1H), 6.73 (d, $J=$ $7.9 \mathrm{~Hz}, 1 \mathrm{H}), 6.63(\mathrm{~d}, J=7.7 \mathrm{~Hz}, 1 \mathrm{H}), 5.86(\mathrm{~s}, 2 \mathrm{H}), 4.35(\mathrm{t}$, $J=6.4 \mathrm{~Hz}, 2 \mathrm{H}), 3.90$ (s, 3H), 3.84 (s, 3H), 3.50 (t, $J=6.3$ $\mathrm{Hz}, 2 \mathrm{H}), 2.30$ (s, 3H); ${ }^{13} \mathrm{C}$ NMR (100 MHz, DMSO- $\left.d_{6}\right) \delta$ : $188.64,163.33,160.43,160.31,153.84,151.10,142.29$, $137.52,130.79,130.48,128.16,124.90,123.72,116.42$, $115.45,106.77,98.85,66.84,56.25,55.99,30.19,10.24$; ESI-HRMS calcd for $\mathrm{C}_{24} \mathrm{H}_{27} \mathrm{O}_{4} \mathrm{~N}_{4} \mathrm{~S}[\mathrm{M}+\mathrm{H}]^{+} 467.17475$, found 467.17401 .

1-(4-(2-((4-氨基-5-甲基-4H-1,2,4-三唑-3-基)硫基) 乙氧基)苯基)-5-(3-硝基苯基)-1,4-戊二烯-3-酮 $\left(\mathbf{F}_{8}\right.$ ): 黄色 固体, 收率: $40 \%$. m.p. $128.4 \sim 130.0{ }^{\circ} \mathrm{C} ;{ }^{1} \mathrm{H}$ NMR $(400$ MHz, DMSO- $\left.d_{6}\right) \delta: 8.63(\mathrm{~s}, 1 \mathrm{H}), 8.28(\mathrm{~d}, J=7.9 \mathrm{~Hz}, 1 \mathrm{H})$, 8.25 (d, $J=7.7 \mathrm{~Hz}, 1 \mathrm{H}), 7.89$ (t, $J=7.1 \mathrm{~Hz}, 1 \mathrm{H}), 7.83$ (d, $J=6.9 \mathrm{~Hz}, 1 \mathrm{H}), 7.79$ (d, $J=6.1 \mathrm{~Hz}, 2 \mathrm{H}), 7.74$ (d, $J=6.0$ $\mathrm{Hz}, 1 \mathrm{H}), 7.55(\mathrm{~d}, J=6.5 \mathrm{~Hz}, 1 \mathrm{H}), 7.22(\mathrm{~d}, J=6.0 \mathrm{~Hz}, 1 \mathrm{H})$, $7.08(\mathrm{~d}, J=7.9 \mathrm{~Hz}, 2 \mathrm{H}), 5.87$ (s, 2H), 4.35 (t, $J=6.4 \mathrm{~Hz}$, $2 \mathrm{H}), 3.50(\mathrm{t}, J=6.3 \mathrm{~Hz}, 2 \mathrm{H}), 2.30(\mathrm{~s}, 3 \mathrm{H}) ;{ }^{13} \mathrm{C}$ NMR $(100$ $\mathrm{MHz}$, DMSO- $\left.d_{6}\right) \quad \delta: 188.73,160.71,153.85,151.11$, $148.83,143.77,140.21,137.21,134.89,131.00,130.91$, 128.63, 127.98, 124.97, 123.97, 123.30, 115.51, 66.87, 30.16, 10.24; ESI-HRMS calcd for $\mathrm{C}_{22} \mathrm{H}_{22} \mathrm{O}_{4} \mathrm{~N}_{5} \mathrm{~S}[\mathrm{M}+\mathrm{H}]^{+}$ 452.13870 , found 452.13766 .

1-(4-(2-((4-氨基-5-甲基-4H-1,2,4-三唑-3-基)硫基) 乙氧基)苯基)-5-(2-氯苯基)-1,4-戊二烯-3-酩 $\left(\mathbf{F}_{9}\right.$ ): 黄色固 体, 收率: $29 \%$. m.p. $155.9 \sim 157.1{ }^{\circ} \mathrm{C} ;{ }^{1} \mathrm{H}$ NMR (400 MHz, DMSO- $\left.d_{6}\right) \delta: 8.05(\mathrm{~d}, J=7.9 \mathrm{~Hz}, 1 \mathrm{H}), 7.95(\mathrm{~d}, J=$ $6.1 \mathrm{~Hz}, 1 \mathrm{H}), 7.86(\mathrm{~d}, J=6.1 \mathrm{~Hz}, 1 \mathrm{H}), 7.75(\mathrm{~d}, J=6.0 \mathrm{~Hz}$, $2 \mathrm{H}), 7.57(\mathrm{~d}, J=6.4 \mathrm{~Hz}, 1 \mathrm{H}), 7.52(\mathrm{t}, J=6.3 \mathrm{~Hz}, 1 \mathrm{H}), 7.47$ (t, $J=6.6 \mathrm{~Hz}, 1 \mathrm{H}), 7.45(\mathrm{~d}, J=6.0 \mathrm{~Hz}, 1 \mathrm{H}), 7.12(\mathrm{~d}, J=7.9$ $\mathrm{Hz}, 1 \mathrm{H}), 7.07$ (d, $J=7.7 \mathrm{~Hz}, 2 \mathrm{H}), 5.87$ (s, 2H), 4.35 (t, $J=$ $6.4 \mathrm{~Hz}, 2 \mathrm{H}), 3.51$ (t, $J=6.3 \mathrm{~Hz}, 2 \mathrm{H}), 2.30(\mathrm{~s}, 3 \mathrm{H}) ;{ }^{13} \mathrm{C}$
NMR (100 MHz, DMSO- $\left.d_{6}\right) \delta: 188.49,160.74,153.85$, $151.10,144.02,137.08,134.61,132.97,132.22,131.03$, $130.54,128.69,128.21,128.19,127.92,124.84,115.53$, 66.87, 30.17, 10.24; ESI-HRMS calcd for $\mathrm{C}_{22} \mathrm{H}_{22} \mathrm{O}_{2} \mathrm{~N}_{4} \mathrm{ClS}$ $[\mathrm{M}+\mathrm{H}]^{+}$441.11465, found 441.11404 .

1-(4-(2-((4-氨基-5-甲基-4H-1,2,4-三唑-3-基)硫基) 乙氧基)苯基)-5-(4-甲氧基苯基)-1,4-戊二烯-3-酮 $\left(\mathbf{F}_{\mathbf{1 0}}\right)$ : 黄色固体, 收率: $62 \%$. m.p. $138.3 \sim 139.9{ }^{\circ} \mathrm{C} ;{ }^{1} \mathrm{H}$ NMR $\left(400 \mathrm{MHz}\right.$, DMSO- $\left.d_{6}\right) \delta: 7.76(\mathrm{~d}, J=6.4 \mathrm{~Hz}, 3 \mathrm{H}), 7.74(\mathrm{~d}$, $J=6.0 \mathrm{~Hz}, 2 \mathrm{H}), 7.71(\mathrm{~d}, J=6.0 \mathrm{~Hz}, 1 \mathrm{H}), 7.22(\mathrm{~d}, J=6.5$ $\mathrm{Hz}, 1 \mathrm{H}), 7.18(\mathrm{~d}, J=6.6 \mathrm{~Hz}, 1 \mathrm{H}), 7.06(\mathrm{~d}, J=7.8 \mathrm{~Hz}, 2 \mathrm{H})$, $7.03(\mathrm{~d}, J=7.7 \mathrm{~Hz}, 2 \mathrm{H}), 5.87$ (s, 2H), 4.34 (t, $J=6.4 \mathrm{~Hz}$, $2 \mathrm{H}), 3.82(\mathrm{~s}, 3 \mathrm{H}), 3.51(\mathrm{t}, J=6.4 \mathrm{~Hz}, 2 \mathrm{H}), 2.29(\mathrm{~s}, 3 \mathrm{H}) ;{ }^{13} \mathrm{C}$ NMR $\left(100 \mathrm{MHz}\right.$, DMSO- $\left.d_{6}\right) \delta: 188.65,161.66,160.47$, $153.86,151.13,142.74,142.55,130.84,130.80,128.13$, $127.83,124.09,124.08,115.44,114.95,66.81,55.84$, 30.11, 10.24; ESI-HRMS calcd for $\mathrm{C}_{23} \mathrm{H}_{25} \mathrm{O}_{3} \mathrm{~N}_{4} \mathrm{~S}[\mathrm{M}+\mathrm{H}]^{+}$ 437.16419, found 437.16360.

1-(4-(2-((4-氨基-5-甲基-4H-1,2,4-三唑-3-基)硫基) 乙氧基)苯基)-5-(4-甲基噻唑-5-基)-1,4-戊二烯-3-酮 $\left(\mathbf{F}_{11}\right)$ : 黄色固体, 收率: $42 \%$. m.p. $144.4 \sim 145.9{ }^{\circ} \mathrm{C} ;{ }^{1} \mathrm{H}$ NMR (400 MHz, DMSO- $\left.d_{6}\right) \delta: 9.12(\mathrm{~s}, 1 \mathrm{H}), 7.89(\mathrm{~d}, J=6.5 \mathrm{~Hz}$, 1H), $7.76(\mathrm{~d}, J=6.0 \mathrm{~Hz}, 2 \mathrm{H}), 7.72(\mathrm{~d}, J=7.9 \mathrm{~Hz}, 1 \mathrm{H}), 7.26$ (d, $J=6.1 \mathrm{~Hz}, 1 \mathrm{H}), 7.06(\mathrm{~d}, J=7.8 \mathrm{~Hz}, 2 \mathrm{H}), 6.91$ (d, $J=$ $7.9 \mathrm{~Hz}, 1 \mathrm{H}), 5.87$ (s, 2H), 4.34 (t, $J=6.4 \mathrm{~Hz}, 2 \mathrm{H}), 3.50$ (t, $J=6.4 \mathrm{~Hz}, 2 \mathrm{H}), 2.57$ (s, 3H), 2.30 (s, 3H); ${ }^{13} \mathrm{C}$ NMR $(100$ MHz, DMSO- $\left.d_{6}\right) \delta$ : $187.84,160.63,156.81,155.35$, $153.85,151.11,143.20,132.03,131.07,129.22,128.15$, $128.02,123.77,115.44,66.85,30.15,15.97,10.24$; ESIHRMS calcd for $\mathrm{C}_{20} \mathrm{H}_{22} \mathrm{O}_{2} \mathrm{~N}_{5} \mathrm{~S}_{2}[\mathrm{M}+\mathrm{H}]^{+}$428.12094, found 428.12033 .

1-(4-(2-((4-氨基-5-甲基-4H-1,2,4-三唑-3-基)硫基) 乙氧基)苯基)-5-(呋喃-2-基)-1,4-戊二烯-3-酮 $\left(\mathbf{F}_{12}\right)$ : 黄色 固体, 收率: $50 \%$. m.p. $150.4 \sim 151.9{ }^{\circ} \mathrm{C} ;{ }^{1} \mathrm{H}$ NMR $(400$ MHz, DMSO- $\left.d_{6}\right) \delta: 7.91(\mathrm{~d}, J=6.5 \mathrm{~Hz}, 1 \mathrm{H}), 7.76(\mathrm{~d}, J=$ $6.0 \mathrm{~Hz}, 2 \mathrm{H}), 7.69$ (d, $J=5.9 \mathrm{~Hz}, 1 \mathrm{H}), 7.60(\mathrm{~d}, J=6.4 \mathrm{~Hz}$, 1H), $7.23(\mathrm{~d}, J=6.2 \mathrm{~Hz}, 1 \mathrm{H}), 7.05$ (d, $J=7.5 \mathrm{~Hz}, 2 \mathrm{H}), 7.01$ (t, $J=7.9 \mathrm{~Hz}, 1 \mathrm{H}), 6.97(\mathrm{~d}, J=7.0 \mathrm{~Hz}, 1 \mathrm{H}), 6.69$ (d, $J=7.9$ $\mathrm{Hz}, 1 \mathrm{H}), 5.88$ (s, 2H), 4.34 (t, $J=6.4 \mathrm{~Hz}, 2 \mathrm{H}), 3.51$ (t, $J=$ $6.4 \mathrm{~Hz}, 2 \mathrm{H}), 2.30(\mathrm{~s}, 3 \mathrm{H}) ;{ }^{13} \mathrm{C}$ NMR (100 MHz, DMSO- $d_{6}$ ) $\delta: 188.15,160.55,153.86,151.51,151.13,146.51,142.79$, $130.97,129.46,128.05,123.92,123.46,117.00,115.42$, 113.53, 66.82, 30.14, 10.24; ESI-HRMS calcd for $\mathrm{C}_{20} \mathrm{H}_{21} \mathrm{O}_{3} \mathrm{~N}_{4} \mathrm{~S}[\mathrm{M}+\mathrm{H}]^{+}$397.13289, found 397.13223.

1-(4-(2-((4-氨基-5-甲基-4H-1,2,4-三唑-3-基)硫基) 
乙氧基)苯基)-5-(3,4-二甲氧基苯基)-1,4-戊二烯-3-酮 $\left(\mathbf{F}_{13}\right)$ : 黄色固体, 收率: $41 \%$. m.p. 101.1 102.3 ${ }^{\circ} \mathrm{C} ;{ }^{1} \mathrm{H}$ NMR (400 MHz, DMSO- $\left.d_{6}\right) \delta: 7.75(\mathrm{~d}, J=6.1 \mathrm{~Hz}, 2 \mathrm{H})$, 7.72 (d, $J=5.5 \mathrm{~Hz}, 1 \mathrm{H}), 7.67$ (d, $J=5.9 \mathrm{~Hz}, 1 \mathrm{H}), 7.41$ (s, $1 \mathrm{H}), 7.33(\mathrm{~d}, J=7.0 \mathrm{~Hz}, 1 \mathrm{H}), 7.25(\mathrm{~d}, J=6.9 \mathrm{~Hz}, 1 \mathrm{H}), 7.20$ (d, $J=6.9 \mathrm{~Hz}, 1 \mathrm{H}), 7.06(\mathrm{~d}, J=7.8 \mathrm{~Hz}, 2 \mathrm{H}), 7.03$ (d, $J=$ $7.6 \mathrm{~Hz}, 1 \mathrm{H}), 5.87$ (s, 2H), 4.34 (t, $J=6.4 \mathrm{~Hz}, 2 \mathrm{H}), 3.85$ (s, $3 \mathrm{H}), 3.82$ (s, $3 \mathrm{H}), 3.51$ (t, $J=6.3 \mathrm{~Hz}, 2 \mathrm{H}), 2.30(\mathrm{~s}, 3 \mathrm{H}) ;{ }^{13} \mathrm{C}$ NMR $\left(100 \mathrm{MHz}\right.$, DMSO- $\left.d_{6}\right) \delta: 188.63,160.47,153.85$, $151.54,151.12,149.46,143.23,142.42,130.82,128.15$, $128.04,124.46,123.97,123.68,115.45,112.12,110.98$, 66.84, 56.08, 56.06, 30.17, 10.24; ESI-HRMS calcd for $\mathrm{C}_{24} \mathrm{H}_{27} \mathrm{O}_{4} \mathrm{~N}_{4} \mathrm{~S}[\mathrm{M}+\mathrm{H}]^{+}$467.17475, found 467.17389.

1-(4-(2-((4-氨基-5-甲基-4H-1,2,4-三唑-3-基)硫基) 乙氧基)苯基)-5-(4-溴苯基)-1,4-戊二烯-3-酮 $\left(\mathbf{F}_{14}\right)$ : 黄色 固体, 收率: $43 \%$. m.p. $185.0 \sim 186.4{ }^{\circ} \mathrm{C} ;{ }^{1} \mathrm{H}$ NMR (400 MHz, DMSO- $\left.d_{6}\right) \delta: 7.79(\mathrm{~d}, J=6.1 \mathrm{~Hz}, 3 \mathrm{H}), 7.73$ (d, $J=$ $5.5 \mathrm{~Hz}, 3 \mathrm{H}), 7.67$ (d, $J=5.8 \mathrm{~Hz}, 2 \mathrm{H}), 7.39$ (d, $J=6.1 \mathrm{~Hz}$, $1 \mathrm{H}), 7.21(\mathrm{~d}, J=6.8 \mathrm{~Hz}, 1 \mathrm{H}), 7.07(\mathrm{~d}, J=7.7 \mathrm{~Hz}, 2 \mathrm{H}), 5.87$ (s, 2H), 4.35 (t, $J=6.4 \mathrm{~Hz}, 2 \mathrm{H}), 3.51(\mathrm{t}, J=6.4 \mathrm{~Hz}, 2 \mathrm{H})$, $2.31(\mathrm{~s}, 3 \mathrm{H}) ;{ }^{13} \mathrm{C}$ NMR $\left(100 \mathrm{MHz}\right.$, DMSO- $\left.d_{6}\right) \delta: 188.74$, $160.63,153.84,151.09,143.36,141.34,134.61,132.42$, $130.94,130.85,128.03,126.89,124.18,124.07,115.50$, 66.87, 30.19, 10.24; ESI-HRMS calcd for $\mathrm{C}_{22} \mathrm{H}_{22} \mathrm{O}_{2} \mathrm{~N}_{4} \mathrm{BrS}$ $[\mathrm{M}+\mathrm{H}]^{+}$485.06414, found 485.06293.

1-(4-(2-((4-氨基-5-甲基-4H-1,2,4-三唑-3-基)硫基) 乙氧基)苯基)-5-(2,4-二氯苯基)-1,4-戊二烯-3-酮 $\left(\mathbf{F}_{\mathbf{1 5}}\right)$ : 黄色固体, 收率: $35 \%$. m.p. $165.1 \sim 166.6{ }^{\circ} \mathrm{C} ;{ }^{1} \mathrm{H}$ NMR $\left(400 \mathrm{MHz}, \mathrm{DMSO}-d_{6}\right) \delta: 8.07(\mathrm{~d}, J=7.8 \mathrm{~Hz}, 1 \mathrm{H}), 7.88(\mathrm{~d}$, $J=6.1 \mathrm{~Hz}, 1 \mathrm{H}), 7.84(\mathrm{~d}, J=6.6 \mathrm{~Hz}, 1 \mathrm{H}), 7.75$ (d, $J=6.1$ $\mathrm{Hz}, 2 \mathrm{H}), 7.73$ (s, 1H), 7.55 (d, $J=6.4 \mathrm{~Hz}, 1 \mathrm{H}), 7.53$ (d, $J=$ $6.4 \mathrm{~Hz}, 1 \mathrm{H}), 7.09$ (d, $J=7.9 \mathrm{~Hz}, 1 \mathrm{H}), 7.06(\mathrm{~d}, J=7.8 \mathrm{~Hz}$, $2 \mathrm{H}), 5.86(\mathrm{~s}, 2 \mathrm{H}), 4.34$ (t, $J=6.4 \mathrm{~Hz}, 2 \mathrm{H}), 3.51$ (t, $J=6.3$ $\mathrm{Hz}, 2 \mathrm{H}), 2.30(\mathrm{~s}, 3 \mathrm{H}) ;{ }^{13} \mathrm{C}$ NMR (100 MHz, DMSO- $\left.d_{6}\right) \delta$ : $188.39,160.78,153.84,151.08,144.20,135.85,135.81$, $135.39,132.08,131.04,130.01,129.93,128.68,128.46$, 127.90, 124.84, 115.55, 66.88, 30.19, 10.24; ESI-HRMS calcd for $\mathrm{C}_{22} \mathrm{H}_{21} \mathrm{O}_{2} \mathrm{~N}_{4} \mathrm{Cl}_{2} \mathrm{~S}[\mathrm{M}+\mathrm{H}]^{+}$475.07568, found 475.07510.

1-(4-(2-((4-氨基-5-甲基-4H-1,2,4-三唑-3-基)硫基) 乙氧基)苯基)-5-(4-甲基苯基)-1,4-戊二烯-3-酮 $\left(\mathbf{F}_{16}\right)$ : 黄 色固体, 收率: $63 \%$. m.p. $169.0 \sim 170.8{ }^{\circ} \mathrm{C} ;{ }^{1} \mathrm{H}$ NMR $\left(400 \mathrm{MHz}, \mathrm{DMSO}-d_{6}\right) \delta: 7.77(\mathrm{~d}, J=6.0 \mathrm{~Hz}, 1 \mathrm{H}), 7.75$ (d, $J=6.1 \mathrm{~Hz}, 2 \mathrm{H}), 7.73(\mathrm{~d}, J=5.5 \mathrm{~Hz}, 1 \mathrm{H}), 7.69$ (d, $J=5.9$ $\mathrm{Hz}, 2 \mathrm{H}), 7.30(\mathrm{~d}, J=6.0 \mathrm{~Hz}, 2 \mathrm{H}), 7.27(\mathrm{~d}, J=6.2 \mathrm{~Hz}, 1 \mathrm{H})$, $7.22(\mathrm{~d}, J=6.0 \mathrm{~Hz}, 1 \mathrm{H}), 7.07$ (d, $J=7.7 \mathrm{~Hz}, 2 \mathrm{H}), 5.87$ (s, 2H), 4.35 (t, $J=6.4 \mathrm{~Hz}, 2 \mathrm{H}), 3.51(\mathrm{t}, J=6.4 \mathrm{~Hz}, 2 \mathrm{H}), 2.36$ (s, 3H), $2.31(\mathrm{~s}, 3 \mathrm{H}) ;{ }^{13} \mathrm{C}$ NMR (100 MHz, DMSO- $\left.d_{6}\right) \delta$ : 188.77, 160.54, 153.84, 151.10, 142.88, 142.78, 140.90, $132.55,130.89,130.07,128.98,128.11,125.34,124.11$, 115.47, 66.86, 30.19, 21.55, 10.24; ESI-HRMS calcd for $\mathrm{C}_{23} \mathrm{H}_{25} \mathrm{O}_{2} \mathrm{~N}_{4} \mathrm{~S}[\mathrm{M}+\mathrm{H}]^{+}$421.16927, found 421.16855 .

\section{3 目标化合物的抑菌活性测试}

采用浊度法, 以柑橘溃疡病菌、水稻白叶枯病菌和 烟草青枯病菌为测试对象, 以叶枯唑为实验对照药剂, 在 100 和 $50 \mu \mathrm{g} / \mathrm{mL}$ 浓度下, 测试目标化合物的体外抑菌 活性 ${ }^{[28]}$.

将柑橘溃疡病菌、水稻白叶枯病菌和烟草青枯病菌 放在营养琼脂培养基(NA, $5.0 \mathrm{~g}$ 蛋白炼, $1.0 \mathrm{~g}$ 酵母粉, 3.0 $\mathrm{g}$ 牛肉膏, $10.0 \mathrm{~g}$ 葡萄糖, $15.0 \mathrm{~g}$ 琼脂和 $1000 \mathrm{~mL}$ 二次水) 固体培养基上培养, 然后置于 $26 \sim 28{ }^{\circ} \mathrm{C}$ 的恒温细菌培 养箱中培养至长出单菌落. 选取适量的黄色单菌落, 将 其放到营养肉汤培养基 $(\mathrm{NB}, 5.0 \mathrm{~g}$ 蛋白胨, $1.0 \mathrm{~g}$ 酵母粉, $3.0 \mathrm{~g}$ 牛肉膏, $10.0 \mathrm{~g}$ 葡萄糖和 $1000 \mathrm{~mL}$ 二次水)液体培养 基中, 在 $26 \sim 28{ }^{\circ} \mathrm{C} 、 180 \mathrm{r} / \mathrm{min}$ 恒温摇床中振荡培养到 对数生长期备用.

将化合物和对照药剂配制为 100 和 $50 \mu \mathrm{g} / \mathrm{mL}$ 浓度, 分别取 $1 \mathrm{~mL}$ 加入到装有 $4 \mathrm{~mL} \mathrm{NB}$ 液体培养基的试管中, 再分别量取 $40 \mu \mathrm{L}$ 含有柑橘溃疡病菌、水稻白叶枯病菌 和烟草青枯病菌的 NB 液体培养基加入试管中, 于 26 $28{ }^{\circ} \mathrm{C} 、 180 \mathrm{r} / \mathrm{min}$ 恒温摇床振荡培养 $24 \mathrm{~h}$. 在分光光度 计上, 测定 $595 \mathrm{~nm}$ 波长处加药无菌 NB 液体培养基的 $\mathrm{OD}_{595}$ 值, 以及测定各个浓度的菌液 $\mathrm{OD}_{595}$ 值.

校正 $\mathrm{OD}_{595}$ 值 $=$ 含菌培养基 $\mathrm{OD}_{595}$-无菌培养基 $\mathrm{OD}_{595}$ 抑制率 $(\%)=$ (校正后对照培养基菌液 $\mathrm{OD}_{595}$ 一校正含药 培养基 $\mathrm{OD}_{595}$ )/校正后对照培养基菌液 OD 值 $\times 100 \%$.

\section{4 扫描电镜成像实验}

量取 $1.5 \mathrm{~mL}$ 生长至对数期的柑橘溃疡病菌 (X. citri) 菌液于 $2 \mathrm{~mL}$ 离心管中, 低速离心 $(6000 \mathrm{r} / \mathrm{min})$ 约 $1 \mathrm{~min}$, 使细菌离心成团, 加入 $1 \mathrm{~mL}$ 磷酸盐缓冲溶液(PBS, $\mathrm{pH}$ $=7.2$ ) 以洗去培养基, 然后再加入 $1.5 \mathrm{~mL}$ PBS 缓冲溶液, 用枪头轻轻重悬，最后加入目标化合物 $\mathbf{F}_{\mathbf{6}}$ 药液使其浓 度分别为 25 和 $50 \mu \mathrm{g} / \mathrm{mL}$, 空白对照补加二甲基亚砜 (DMSO) $10 \mu \mathrm{L}$, 室温孵育 $8 \sim 10 \mathrm{~h}^{[24]}$.

吸取捊育后菌液 $1 \mathrm{~mL}$ 于 $2 \mathrm{~mL}$ 离心管中低速离心 (6000 r/min)约 $1 \mathrm{~min}$, 使细菌离心成团, 弃去上清液, 沉 淀菌体用 $1 \mathrm{~mL}$ PBS 溶液洗涤 3 次以洗去药剂; 加入 1 $\mathrm{mL} 2.5 \%$ 戊二醛固定液固定 $8 \sim 10 \mathrm{~h}$ 或过夜 $\left(4{ }^{\circ} \mathrm{C}\right)$, 弃去 固定液, 再用 $1 \mathrm{~mL}$ PBS 溶液洗涤 3 次以洗去戊二醛; 菌 


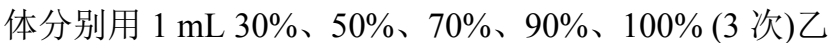
醇依次进行脱水置换(每次置换约 $10 \sim 15 \mathrm{~min}$ ), 脱水后 样品置于冷冻干燥机冷冻干燥 $2 \mathrm{~h}$; 干燥菌体粉末经粘 样、喷金后进行扫描电镜(Nova Nano SEM 450)测试.

辅助材料(Supporting Information) 中间体和目标化 合物的 ${ }^{1} \mathrm{H} N \mathrm{NMR},{ }^{13} \mathrm{C} \mathrm{NMR}, \mathrm{HRMS}$ 谱图. 这些材料可以 免费从本刊网站(http://sioc-journal.cn/)上下载.

\section{References}

[1] Chen, L.-J.; Li, P.; Wang, X.-B.; Ruan, X.-H.; Xue, W. Chem. Bull. 2017, 80, 1156 (in Chinese) (陈丽娟, 李普, 王晓斌, 阮祥辉, 薛伟, 化学通报, 2017, 80, 1156.)

[2] Ren, Y. H.; Jin, H.; Tao, K.; Hou, T. P. Mol. Cell. Toxicol. 2015, 11, 187.

[3] Peron, F.; Lazarin-Bidóia, D.; Din, Z. U.; Rodrigues-Filho, E.; Ueda-Nakamura, T.; Silva, S. O.; Nakamura, C. V. Biomed. Res. Int. 2017, 1

[4] Wu, J.; Zhu, Y. Y.; Zhao, Y. H.; Shan, W. L.; Hu, D. Y.; Chen, J. X. Chin. Chem. Lett. 2016, 27, 948

[5] Chen, C. L.; Chen, J.; Gu, H. Y.; Bao, N.; Dai, H. Molecules 2017, $22,1126$.

[6] Zhou, J.; Tao, Q. Q.; Wang, P. Y.; Shao, W. B.; Wu, Z. B.; Li, Z. Bioorg. Med. Chem. Lett. 2018, 28, 1742.

[7] Zhu, H. P.; Xu, T. T.; Qiu, C. Y.; Wu, B. B.; Zhang, Y. L.; Chen, L. F. Eur. J. Med. Chem. 2016, 121, 181.

[8] Wang, Z. S.; Chen, L. Z.; Zhou, H. P.; Liu, X. H.; Chen, F. H. Bioorg. Med. Chem. Lett. 2017, 27, 1803.

[9] Luo, H.; Yang, S. J.; Cai, Y. Q.; Peng, Z. J.; Liu, T. Eur. J. Med. Chem. 2014, 84, 746.

[10] Badr, G.; Gul, H. I.; Yamali, C.; Mohamed, A. A. M.; Badr, B. M.; Gul, M. Bioorg. Chem. 2018, 78, 46.

[11] Luo, H.; Yang, S. J.; Hong, D.; Xue, W.; Xie, P. Chem. Cent. J. 2017, 11, 23.

[12] Xue, W.; Gong, H.-Y.; Qiu, Q.-J.; Zhao, H.-J.; Li, H.-C.; Han, F.-F. Chem. Reag. 2013, 35, 201 (in Chinese).

(薛伟, 龚华玉, 仇秋娟, 赵洪菊, 李海畅, 韩菲菲, 化学试剂, 2013, 35, 201.)
[13] Zhang, J.-P.; Li, P.; Wang, Y.-H.; Zhang, C.; Chen, L.-J.; Tang, X.; He, M.; Xue, W. Chem. J. Chin. Univ. 2018, 39, 1455 (in Chinese). (张菊平, 李普, 王一会, 张橙, 陈丽娟, 汤旭, 贺鸣, 薛伟, 高 等学校化学学报, 2018, 39, 1455.)

[14] Kulabaş, N.; Tatar, E.; Özakpınar, Ö. B.; Özsavcı, D.; Pannecouque, C.; Clercq, E. D.; Küçükgüzel, İ. Eur. J. Med. Chem. 2016, 121, 58.

[15] Somagond, S. M.; Kamble, R. R.; Kattimani, P. P.; Shaikh, S. K. J.; Dixit, S. R.; Joshi, S. D. ChemistrySelect 2018, 3, 2004.

[16] Wang, X. B.; Zhong, X. M.; Zhu, X. S.; Wang, H.; Li, Q.; Zhang, J. P.; Xue, W. Chem. Pap. 2017, 71, 1953.

[17] Zhai, Z.-W.; Wang, Q.; Shen, Z.-H.; Tan, C.-X.; Weng, J.-Q.; Liu, X.-H. Chin. J. Org. Chem. 2017, 37, 232 (in Chinese) (翟志文, 汪乔, 沈钟华, 谭成侠, 翁建全, 刘幸海, 有机化学, 2017, 37, 232.)

[18] Başaran, E.; Karaküçük-Iyidoğan, A.; Schols, D.; Oruç-Emre, E. E. Chirality 2016, 28, 495.

[19] Jin, R. Y.; Liu, J. L.; Zhang, G. H.; Li, J. J.; Zhang, S.; Guo, H Chem. Biodiversity 2018.

[20] Xu, F. Z.; Shao, J. H.; Zhu, Y. Y.; Liu, L. W.; Zhao, Y. H.; Shan, W. L. Chem. Pap. 2017, 71, 729 .

[21] Du, H.; Fan, Z.-J.; Yang, L.; Bao, X.-P. Chin. J. Org. Chem. 2018, 38,531 (in Chinese). (杜欢，范治江，杨岗，鲍小平，有机化学, 2018, 38, 531.)

[22] Yan, B.-R.; Lü, X.-Y.; Du, H.; Bao, X.-P. Chin. J. Org. Chem. 2016, 36, 207 (in Chinese). (闵柏任，吕新阳，杜欢，鲍小平，有机化学, 2016, 36, 207.)

[23] Lin, G.-S.; Chen, Z.-C.; Duan, W.-G.; Wang, X.-Y.; Lei, F.-H. Chin. J. Org. Chem. 2018, 38, 2085 (in Chinese). (林桂汕，陈智聪，段文贵，王晓宇，雷福厚，有机化学，2018, 38, 2085.)

[24] Zhou, J. M.S. Thesis, Guizhou University, Guiyang, 2018 (in Chinese). (周建，硕士论文，贵州大学，贵阳, 2018.)

[25] Liu, C.-Y.; Zhao, Q.-Q.; Li, J. Chem. Reag. 2001, 23, 344 (in Chinese). (柳翠英, 赵全芹, 李娟, 化学试剂, 2001, 23, 344.)

[26] Gan, X. H.; Hu, D. Y.; Li, P.; Wu, J.; Chen, X. W.; Xue, W.; Song, B. A. Pest Manage. Sci. 2015, 72, 534.

[27] Wu, F.; Li, P.; Hu, D. Y.; Song, B. A. Res. Chem. Intermed. 2016, $42,7153$.

[28] Xu, W. M.; Han, F. F.; He, M.; Hu, D. Y.; He, J.; Yang, S.; Song, B. A. J. Agric. Food Chem. 2012, 60, 1036. 